\title{
Clinical evaluation of Bladder CARE, a new epigenetic test for bladder cancer detection in urine samples
}

\author{
Paolo Piatti', Yap Ching Chew ${ }^{1}$, Michiko Suwoto', Taikun Yamada' ${ }^{1}$, Benjamin Jara ${ }^{1}$, Xi-Yu Jia ${ }^{1,2}$, Wei Guo', \\ Saum Ghodoussipour ${ }^{3}$, Siamak Daneshmand ${ }^{3}$, Hamed Ahmadi ${ }^{3}$, Jeffrey Rice ${ }^{1}$, Jeffrey Bhasin ${ }^{1}$, Faith Holloway ${ }^{1}$, \\ Yvonne Tsai ${ }^{3}$, Yoshitomo Chihara ${ }^{3}$ and Gangning Liang ${ }^{3^{*}}$ (D)
}

\begin{abstract}
Background: Bladder cancer (BC) is the 5th most common cancer in the USA. Non-muscle invasive bladder cancer represents about $70 \%$ of all cases and has generally a favorable outcome. However, recurrence rates as high as 60 to $70 \%$ and progression rates of 10 to $20 \%$ necessitate intensive surveillance with cystoscopy. The invasiveness and high cost of cystoscopy poses significant burden on BC patients as well as on the healthcare system. In this study we test the feasibility of a simple, sensitive, and non-invasive detection of BC using Bladder CARE test in urine samples.

Results: Urine from 136 healthy and $77 \mathrm{BC}$ subjects was collected using the at-home Bladder CARE Urine Collection Kit and analyzed with Bladder CARE test. The test measures the methylation level of three BC-specific biomarkers and two internal controls using methylation-sensitive restriction enzymes coupled with qPCR. Bladder CARE showed an overall sensitivity of $93.5 \%$, a specificity of $92.6 \%$, and a PPV and NPV of $87.8 \%$ and $96.2 \%$, respectively. Bladder CARE has an LOD as low as $0.046 \%$, which equates to detecting 1 cancer cell for every 2,200 cells analyzed. We also provided evidence that bisulfite-free methods to assess DNA methylation, like Bladder CARE, are advantageous compared to conventional methods that rely on bisulfite conversion of the DNA.

Conclusion: Highly sensitive detection of $B C$ in urine samples is possible using Bladder CARE. The low LOD of the test and the measurement of epigenetic biomarkers make Bladder CARE a good candidate for the early detection of $B C$ and possibly for the routine screening and surveillance of BC patients. Bladder CARE and the at-home urine sample collection system have the potential to (1) reduce unnecessary invasive testing for BC (2) reduce the burden of surveillance on patients and on the healthcare system, (3) improve the detection of early stage BC, and (4) allow physicians to streamline the monitoring of patients.
\end{abstract}

Keywords: Bladder cancer, DNA methylation, At-home sample collection, Non-invasive testing, Urine

\section{Background}

Bladder cancer $(\mathrm{BC})$ is the 5 th most common cancer in the USA [1] with 81,400 new cases and 17,980 deaths estimated for 2020 and an incidence rate of $2.4 \%$ [2]. BC

*Correspondence: gliang@usc.edu

${ }^{3}$ Department of Urology, Keck School of Medicine, University of Southern California, Los Angeles, CA, USA

Full list of author information is available at the end of the article occurs more frequently in Caucasians compared to other ethnicities, and it is three to four times more frequent in men than in women, making it the 4th most common cancer in men in the USA and the 8th most common cause of cancer death [1-3]. Tobacco smoking is the primary risk factor for $\mathrm{BC}$, and it is estimated to contribute to the development of up to $50 \%$ of all bladder tumors. Other well-documented risk factors include chronic urinary tract infections, occupational exposure to original author(s) and the source, provide a link to the Creative Commons licence, and indicate if changes were made. The images or other third party material in this article are included in the article's Creative Commons licence, unless indicated otherwise in a credit line to the material. If material is not included in the article's Creative Commons licence and your intended use is not permitted by statutory regulation or exceeds the permitted use, you will need to obtain permission directly from the copyright holder. To view a copy of this licence, visit http://creativecommons.org/licenses/by/4.0/. The Creative Commons Public Domain Dedication waiver (http://creativeco mmons.org/publicdomain/zero/1.0/) applies to the data made available in this article, unless otherwise stated in a credit line to the data. 
carcinogens (e.g., aromatic amines, polycyclic aromatic hydrocarbons), chronic exposure to arsenic, pelvic radiation, and genetic predisposition [4-9]. The most common histology is urothelial carcinoma; approximately $70 \%$ of all cases are non-muscle invasive bladder cancer (NMIBC), while the remaining $30 \%$ are represented by muscle invasive bladder cancer (MIBC) [10, 11]. NMIBC has a generally favorable outcome after transurethral resection of bladder tumor (TURBT; 80\% 10-year survival). However, recurrence rates as high as 60 to $70 \%$ and progression rates of 10 to $20 \%$ necessitate intensive surveillance for NMIBC. Current guidelines recommend lifelong surveillance with cystoscopy [12-14]. While cystoscopy is the gold standard for the detection of bladder tumors, it is invasive, costly, and poses significant burden on the patient and on the healthcare system. The costs of treatment and surveillance make $\mathrm{BC}$ the most expensive cancer to manage [15].

In addition to cytology, a number of tests for the non-invasive detection of $\mathrm{BC}$ have been developed including Nuclear Matrixprotein-22 (NMP22) [16], the ImmunoCyt assay (Scimedx, Denville, NJ, USA) [17], Bladder Tumor-associated Antigen (BTA) [18], the UroVysion (Abbott Molecular Inc., Des Plaines, IL, USA) [19], Cxbladder [20], UroMark [21], and EpiCheck [22]. The adoption of some of these tests in the clinical practice for the detection of $\mathrm{BC}$ is becoming a reality, and it is currently being extensively evaluated. A summary of the performance characteristics of different non-invasive $\mathrm{BC}$ tests in comparison with cytology and cystoscopy is shown in Table 1 [21, 23-28].

DNA methylation is an epigenetic mark that is often altered in many human diseases including cancer $[29,30]$, and it is believed that alterations in DNA methylation are early events in tumorigenesis [11, 31]. DNA methylation, therefore, promises to be a good biomarker for early tumor detection, especially when multiple methylation biomarkers are combined in a multiplex panel [11]. In patients affected by BC, tumor DNA and cells are released in the urine, allowing for the non-invasive detection of cancer-specific methylation biomarkers from urine specimens for both diagnostic and monitoring purposes as described in pioneering works [11,32-35]. DNA methylation is commonly analyzed by treating DNA with sodium bisulfite and then detected using next-generation sequencing and PCR-based approaches [36, 37]. DNA conversion with sodium bisulfite, however, introduces DNA strand breaks and results in the degradation of up to $84-96 \%$ of the input DNA [38], with a consequent reduction of the number of DNA molecules that can be effectively analyzed. This problem is particularly pronounced in samples that contain low or already highly fragmented DNA (e.g., urine DNA and cell-free DNA).

In this study we evaluated the feasibility of Bladder CARE (Pangea Laboratory LLC, CA, USA) in the detection of $B C$ in urine samples. Bladder CARE is a newly developed test that uses methylation-sensitive restriction enzymes to analyze the methylation level of three BC-specific methylation biomarkers (TRNA-Cys, SIM2, and NKX1-1). These loci are hypermethylated in both NMIBC and MIBC and were discovered and validated in previous studies [39]. In the current study we aim to determine the clinical performance of Bladder CARE on a cohort of healthy controls and patients with $\mathrm{BC}$, evaluate the advantages of bisulfite-free methods for the assessment of DNA methylation, and test the feasibility of at-home sample collection.

Table 1 Performance characteristics of different non-invasive BC tests

\begin{tabular}{llll}
\hline Test name & Principle & Sensitivity\% & Specificity\% \\
\hline Bladder CARE & Multiplex MSRE-qPCR & $94^{*}$ & $93^{*}$ \\
BTA stat & Immunoassay & $61[23]$ & $78[23]$ \\
Cxbladder & RT-qPCR & $91[24]$ & Not reported \\
EpiCheck & Multiplex MSRE-qPCR & $63[25,26]$ & $86[25,26]$ \\
ImmunoCyt & Immunocytochemical assay & $62[23]$ & $79[23]$ \\
NMP22 BladderChek & Immunoassay & $58[23]$ & $85[23]$ \\
UroMark & Targeted bisulfite sequencing & $98[21]$ & $97[21]$ \\
UroVysion & Fluorescence in situ hybridization (FISH) & $72[27]$ & $83[27]$ \\
Cytology & & $48[23]$ & $86[23]$ \\
Cystoscopy (blue light) & & $92[28]$ & $48[28]$ \\
Cystoscopy (white light) & & $81[28]$ & $49[28]$ \\
\hline
\end{tabular}

Test type and performance characteristics of several non-invasive BC tests are summarized and compared to cytology and cystoscopy techniques

* Performance of Bladder CARE is based on the results reported in this manuscript 


\section{Results}

\section{Study population cohort demographics}

The study population included a total of 213 subjects. In total, 77 urine samples were collected from individuals affected by $\mathrm{BC}$ (cancer cohort) and 136 from healthy subjects (control cohort). The characteristics of the study population are represented in Table 2.

The cancer cohort had an average age of 66.4 years, was $84.4 \%$ male, and $96.1 \%$ Caucasian. In total, $13 \%$ of the $\mathrm{BC}$ samples were collected from subjects affected by low-grade NMIBC tumor, while $66.2 \%$ from individuals affected by high-grade urothelial carcinoma (41 NMIBC and 10 MIBC). Grading information was not available for the remaining $20.8 \%$ of the samples.

The control cohort had an average age of 50.4 years and was $52.9 \%$ male. The cohort was $73.5 \%$ Caucasian, 16.2\% Asian, 2.2\% African-American, and 0.7\% Hispanic. No ethnicity information was available for the remaining $7.4 \%$ of the cohort.

Table 2 Clinicopathological characteristics of the cohort included in the study $(n=213)$

\begin{tabular}{lll}
\hline Characteristic & $\begin{array}{l}\text { Control cohort } \\
n=136\end{array}$ & $\begin{array}{l}\text { Cancer cohort } \\
n=77\end{array}$ \\
\hline $\begin{array}{l}\text { Age (years) } \\
\text { Mean (range) }\end{array}$ & $50.4(23-88)$ & $66.4(47-86)$ \\
Sex, no. (\%) & & \\
Male & $72(52.9)$ & $65(84.4)$ \\
Female & $64(47.1)$ & $12(15.6)$ \\
Ethnicity, no. (\%) & & \\
Caucasian & $100(73.5)$ & $74(96.1)$ \\
Asian & $22(16.2)$ & $2(2.6)$ \\
African-American & $3(2.2)$ & $0(0)$ \\
Hispanic & $1(0.7)$ & $1(1.3)$ \\
Other & $0(0)$ & $0(0)$ \\
Not available & $10(7.4)$ & $0(0)$ \\
Tumor type, no. (\%) & & \\
NMIBC, TCC & n.a & $66(85.7)$ \\
MIBC, TCC & n.a & $10(13.0)$ \\
AC & n.a & $1(1.3)$ \\
SCC & n.a & $0(0)$ \\
Tumor grade, no. (\%) & & $10(13.0)$ \\
Low grade & n.a & $51(66.2)$ \\
High grade & n.a & $16(20.8)$ \\
Not Available & n.a & \\
\hline NMBC non-musce & & \\
& &
\end{tabular}

NMIBC non-muscle invasive bladder cancer, $M I B C$ muscle invasive bladder cancer, TCC transitional cell carcinoma, $A C$ adenocarcinoma, SCC squamous cell carcinoma, n.a. not applicable
Bladder CARE correctly classifies the cohorts included in the study population

Bladder CARE results between the cancer and the control cohorts were compared. Bladder CARE results are expressed as the Bladder CARE Index (BCI). BCI values are calculated by integrating the methylation level of the three $\mathrm{BC}$ biomarkers and the two internal controls in a proprietary algorithm developed by Pangea Laboratory $[39,40]$.

Based on BCI values, samples are categorized as 'Negative,' 'High-Risk,' and 'Positive.' Specifically, samples with $\mathrm{BCI}<2.5$ are considered Negative for the presence of $\mathrm{BC}$, while samples with $\mathrm{BCI}$ between 2.5 and 5 , and $>5$ are classified as High-Risk and Positive, respectively ([40], Fig. 1).

We observed significantly higher $\mathrm{BCI}$ values for $\mathrm{BC}$ patients compared to $\mathrm{BC}$-free volunteers (average $\mathrm{BCI}$ value for the cancer and control cohorts was 170.1 and 1.3 , respectively; $p=6.2 \times 10^{-7}$; Fig. 2 a). Calculated $95 \%$ CIs for the cancer and the control cohorts were 84.39 to 255.61 and 1.173 to 1.487 , respectively. As shown in Fig. $2 \mathrm{~b}$ the ROC curve AUC was 0.971 ( 1 being perfect discrimination, 0.5 being no better than chance).

\section{Bladder CARE has high sensitivity, specificity, and predictive values}

To evaluate the sensitivity of Bladder CARE we analyzed 77 urine samples collected from the cancer cohort. Five cancer subjects resulted Negative (falsenegative calls), while the other 72 were classified as High-Risk $(n=12)$ and Positive $(n=60)$ by Bladder CARE (Table 4A). Samples resulting High-Risk and Positive were grouped together as positive for the calculation of the overall test sensitivity, which resulted in $93.5 \%$ (94.1\% and $90.0 \%$ for high- and low-grade tumors, respectively. Tables 3, 4A, B). We did not observe a significant difference in the average $\mathrm{BCI}$ values between low-grade and high-grade cancer subcohorts (156.5 and 207.6, respectively; $p=0.7310$ ).

To gain information about the specificity of Bladder CARE, urine samples of 136 healthy individuals were analyzed. Bladder CARE has an overall specificity of 92.6\% (Table 3). Ten false-positive cases (9 High-Risk and 1 Positive) with an average BCI of 3.6 (range 2.75.2) were detected within the control cohort (Table 5A). When we classified the control population based on sex, age, or ethnicity (Table $5 \mathrm{~B}$ ), we did not observe significant differences in $\mathrm{BCI}$; however, we noticed that the majority of the false-positive cases were found among male individuals ( 7 out of 10), between 50 and 80 years old ( 9 out of 10) and Caucasian ( 9 out of 10). 


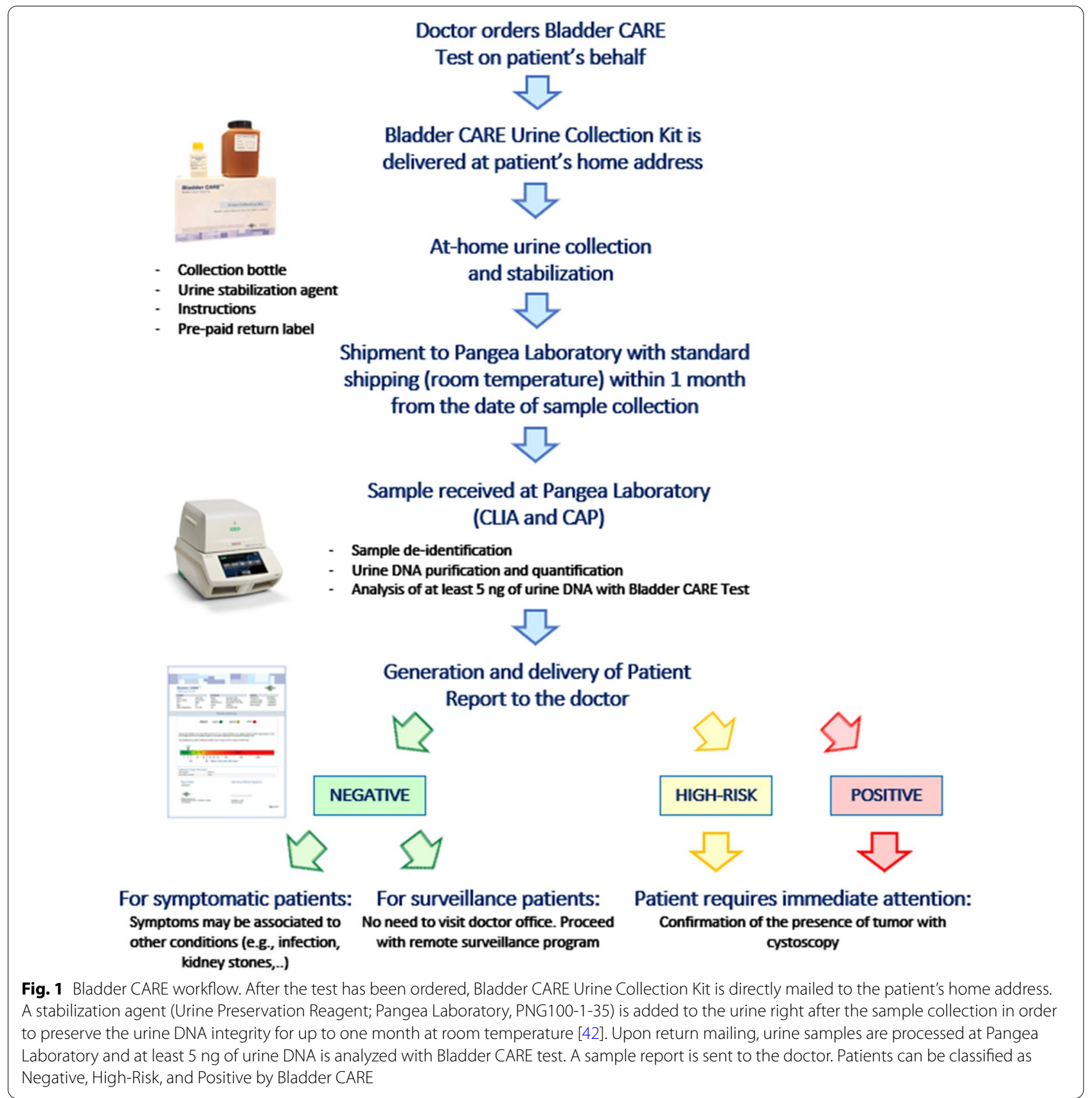

Positive and negative predictive values (PPV and NPV) for Bladder CARE were $87.8 \%$ and $96.2 \%$, respectively (Table 3 ).

\section{Bladder CARE test results can estimate cancer probability} Patients are classified as Negative, High-Risk, or Positive by Bladder CARE. This classification already helps doctors to identify which patients require immediate attention; however, a precise estimation of cancer probability may add another layer of information that can help doctor's decision, especially for patients with High-Risk results.

To provide a continuous estimated probability of cancer, a logistic regression model was generated utilizing the $\mathrm{BCI}$ values and clinical information available for the cancer and control cohorts (Fig. 3). The resulting model had an $F_{1}$-Score of 0.9 (a measure of binary classification accuracy on a $0-1$ scale, with 1 representing perfect precision and recall) and a receiver operating characteristics (ROC) area under the curve (AUC) score of 0.98 

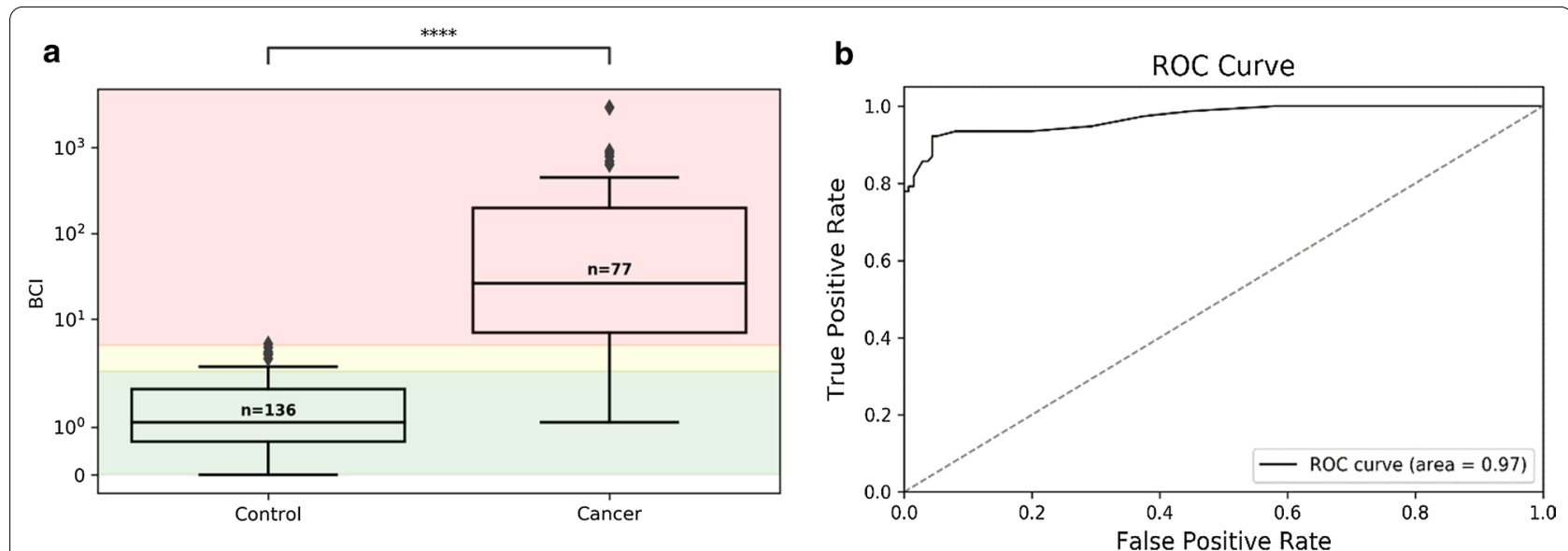

Fig. 2 Bladder CARE test correctly classifies control and cancer cohorts. a Distribution box plots of BCI values (Y-axis) represented on a symmetrical $\log$ scale ( $Y$-axis is linear between 0 and $2 ; Y$-axis $>2$ is illustrated on a base 10 logarithmic scale). The size of each cohort is indicated above each median. Statistical significance (calculated using Student's two-tailed $t$ test) between cohorts is indicated on the top of the figure. Interquartile range (the range of samples from the 25th to 75 th percentile) is represented by the box, and the cohort median value is represented by the horizontal line within the box. Outliers (values outside the minimum and maximum represented by the whiskers, equaling the 25th or 75 th percentile $+1.5 *$ the interquartile range) are indicated by black diamonds. Bladder CARE-negative samples $(\mathrm{BCl}<2.5)$ are delimited by the green area, while High-Risk and Positive samples ( $\mathrm{BCl}$ between 2.5 and 5 , and $>5$ ) are delimited by the yellow and red areas, respectively. b Receiver operating characteristic (ROC) curve using the $\mathrm{BCl}$ value for classification. The area under the curve (AUC) of the ROC curve is a measure of classification performance, plotting the difference in true-positive rate (sensitivity) and false-positive rate (1—specificity) as the classification decision boundary is changed (what value is required to classify as positive), where $0.5=$ no discrimination and $1=$ perfect discrimination. Classification thresholds were taken at a $0.25 \mathrm{BCl}$ interval, with a resulting AUC of 0.971

Table 3 Bladder CARE test performance

\begin{tabular}{ll}
\hline Total cohort $(n=213)$ & \\
\hline True positive & 72 \\
True negative & 126 \\
False positive & 10 \\
False negative & 5 \\
Sensitivity\% & 93.5 \\
Specificity\% & 92.6 \\
PPV\% & 87.8 \\
NPV\% & 96.2
\end{tabular}

Performance of Bladder CARE test calculated from the analysis of the control $(n=136)$ and cancer $(N=77)$ cohorts included in this study

(Additional file 1: Figure S1). The AUC score is a measure of model performance when considering model output as a classifier (on a $0.5-1$ scale, with $\mathrm{AUC}=1$ signifying perfect discrimination).

A plot estimated probability of cancer versus BCI value is shown in Fig. 3a, compared to histogram plots highlighting the distribution of the cancer and control cohorts by BCI value (Fig. 3b, c). The model indicates a low estimated cancer probability for BCI values below 2.5 and highlights a sharp increase in cancer probability into the High-Risk classification with an estimated cancer probability of $80 \%$ at a BCI value of 5 (Fig. 3a).
Figure 3b, c shows that the majority (92.6\%) of the control subjects were classified as such, while most of cancer subjects were classified as Positive and High-Risk (77.9\% and $15.6 \%$, respectively).

\section{Bisulfite-free methods maximize qPCR signal}

Bisulfite treatments to study DNA methylation cause significant sample degradation [31, 38]. In contrast, bisulfite-free approaches like Bladder CARE are believed to minimize the loss of DNA [31], potentially contributing to the overall test sensitivity.

To validate this hypothesis, we analyzed 6 spike-in samples containing different amounts of artificially methylated and untreated blood DNA with methylation-sensitive restriction enzymes (MSRE) qPCR (a bisulfite-free method comparable to Bladder CARE) and with methylation-specific (MS) qPCR method (a common bisulfite-based method). The genomic region selected for this experiment was the CpG island of the human MGMT gene, which is unmethylated in blood DNA samples as shown in Additional file 2: Table S1. Besides the treatment (enzyme digestion or bisulfite conversion) the other variables (primer binding sites, qPCR conditions, and input DNA used) were kept constant between the two experiments. As shown in Fig. 4, despite starting from the same amount of DNA, we 
Table 4 Sensitivity of Bladder CARE for the cancer cohort

(A)

\section{Cancer cohort $(n=77)$}

True positive (Positive + High-Risk)

False negative

$72(60+12)$

Total sensitivity $\%$

5

93.5

(B)

\section{Tumor grade}

Low grade

\section{True positive}

9

48

High grade

Grade not specified

15

\section{False negative}

Sensitivity \%

94.1

93.8

(A) Sensitivity of Bladder CARE calculated for the cancer cohort $(n=77)$, and (B) for the cancer cohort classified based on tumor grade

Table 5 Sensitivity of Bladder CARE

(A)

\section{Control cohort $(n=136)$}

$\begin{array}{ll}\text { True negative } & 126 \\ \text { False positive } & 10 \\ \text { Total specificity \% } & 92.6\end{array}$

(B)

\section{True negative}

False positive

\section{Ethnicity}

\begin{tabular}{|c|c|c|}
\hline Caucasian & 91 & 9 \\
\hline Asian & 22 & 0 \\
\hline African-American & 3 & 0 \\
\hline Hispanic & 1 & 0 \\
\hline Other & n.a & n.a \\
\hline Not available & 9 & 1 \\
\hline \multicolumn{3}{|l|}{ Sex } \\
\hline Male & 65 & 7 \\
\hline Female & 61 & 3 \\
\hline \multicolumn{3}{|l|}{ Age } \\
\hline $20-29$ & 19 & 0 \\
\hline $30-39$ & 30 & 1 \\
\hline $40-49$ & 12 & 0 \\
\hline $50-49$ & 23 & 3 \\
\hline $60-69$ & 24 & 3 \\
\hline $70-79$ & 16 & 3 \\
\hline $80-89$ & 2 & 0 \\
\hline
\end{tabular}

(A) Specificity of Bladder CARE calculated for the control cohort $(n=136)$. (B) Distribution of the false-positive cases based on the cohort classification accordingly to ethnicity, sex, and age; n.a., data not available 
Fig. 3 Correlation between $\mathrm{BCl}$ and probability of being affected by $B C$. a Probability of bladder cancer ( $Y$-axis) based on $B C l$ values ( $X$-axis) as determined by the logistic regression model. b, c Sample frequencies by their $\mathrm{BCl}$ value as histogram plots for the control and cancer cohorts. Cohort $\mathrm{BCl}$ values are divided into 23 bins, divided evenly on a base 10 logarithmic scale from 1 to the max $B C l$ value for 19 bins, with four additional bins representing $B C I$ values from 0 to 1 at 0.25 increments. $\mathrm{BCl}$ values (X-axis) are represented using a symmetrical log scale $(\mathrm{BCl}$ values between 0 and 1 are represented on a linear scale, while values $>2$ are illustrated on a base 10 logarithmic scale). Bladder CARE classifications are delimited by shading, with Negative $(\mathrm{BCl}<2.5)$ as green, and High-Risk $(\mathrm{BCl} 2.5-5)$ and Positive classifications $(\mathrm{BCl}>5)$ delimited by the yellow and red areas, respectively

found that samples analyzed with MSRE-qPCR amplify on average 2.83 PCR cycles earlier compared to the same samples analyzed with MS-qPCR. This corresponds to a substantial gain in qPCR signal $(\sim 86 \%)$ for samples digested with methylation-sensitive restriction enzymes compared to bisulfite-treated samples.

\section{Bladder CARE can detect 1 cancer cell for every 2,200 normal cells}

To gain information about the limit of detection (LOD) of Bladder CARE, we submitted to Pangea Laboratory a set of 12 artificial samples (Additional file 2: Table S2) containing different proportions of LD583 BC cell line DNA [41] and blood DNA isolated from a healthy donor. Blood DNA was used as "background DNA" since Bladder CARE markers are not methylated in blood [39]. Results in Fig. 5 indicate that BCI values decrease linearly with a decrease in the number of cancer cells present in the samples until a concentration of $0.14 \%$. BCI differences between consecutive samples remain significant until sample 8, indicating that Bladder CARE significantly detects the presence of cancer cells at a minimum concentration (LOD) of $0.046 \%$ ( $p=0.031$; Fig. 5 and Additional file 2: Table S2). This is the equivalent of detecting 1 cancer cell in a sample containing 2200 normal cells. Below this limit BCI values do not significantly deviate from the control sample 12 ( $0 \%$ of cancer cell content; Fig. 5 and Additional file 2: Table S2). We must point out, however, that Bladder CARE classifies as High-Risk and Positive in samples with a BCI greater than 2.5. This number corresponds to an LOD between 0.14 and $0.41 \%$ (Fig. 5, Additional file 2: Table S2). Therefore, while the LOD of Bladder CARE can be as low as $0.046 \%$, the LOD of the test in the current setup is between 0.14 and $0.41 \%$.
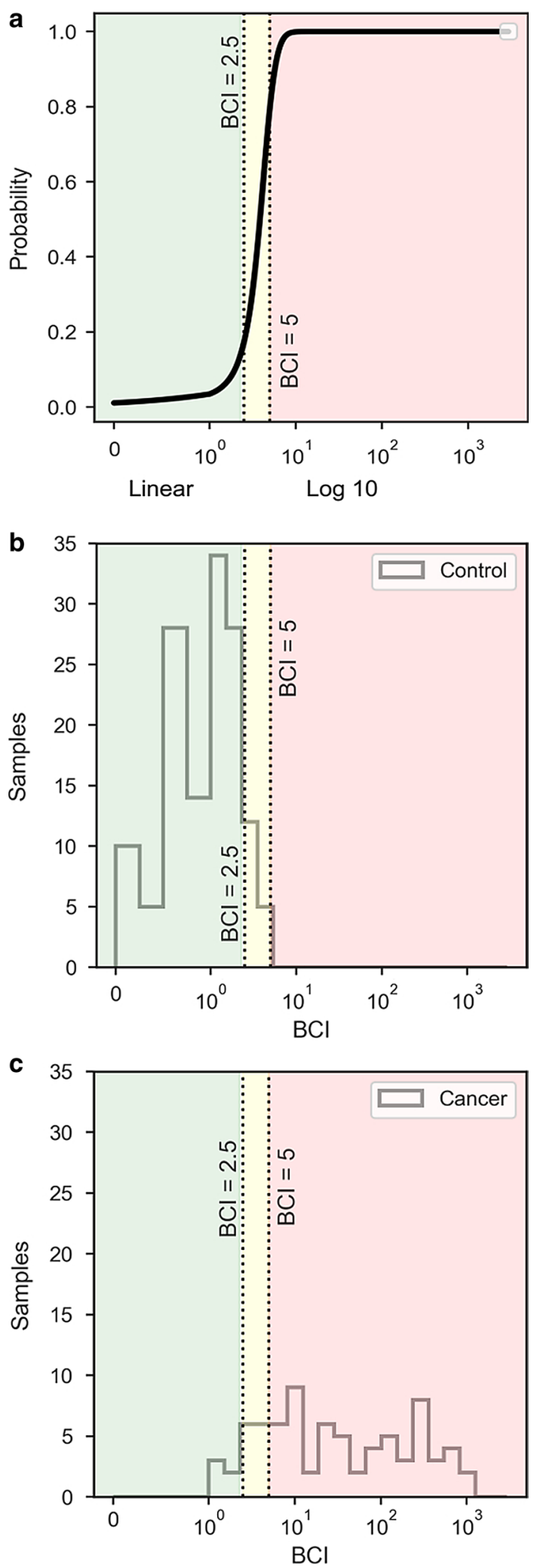


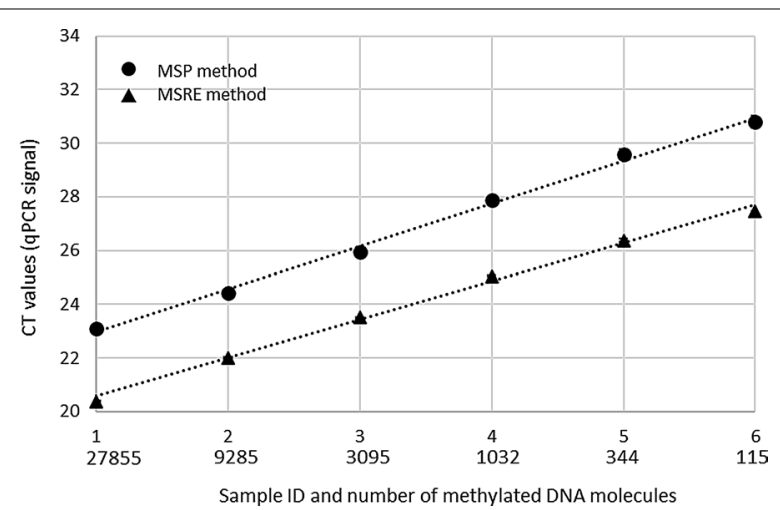

Fig. 4 Comparison between signal generated from MSRE-qPCR and MS-qPCR. The methylation level of the $\mathrm{CpG}$ island of the human MGMT gene was analyzed in a set of 6 spike-in samples containing different amount of artificially methylated and unmethylated DNA. Signals originated from MSRE-qPCR and MS-qPCR are marked by black rectangles and black circles, respectively. Cycle threshold (CT) values are indicated on the $Y$-axis. Sample IDs and calculated methylated DNA copy number (considering that one DNA molecule weighs $3.59 \mathrm{pg}$ [43]) originally present in the samples before restriction digestion or bisulfite conversion is indicated on the $X$-axis. Each sample was tested using $100 \mathrm{ng}$ of spike-in DNA in three technical replicates

\section{Discussion}

The measurement of epigenetic tumor markers in urine specimens represents a non-invasive alternative to detect the presence of $\mathrm{BC}$. Alterations in DNA methylation are believed to be early events in tumorigenesis [14]; therefore, epigenetic tumor biomarkers promise to be good candidates for the early cancer detection of BC.

In this study we tested the performance of a new noninvasive and bisulfite-free epigenetic test for $\mathrm{BC}$ detection, Bladder CARE, and the feasibility of at-home urine sample collection (Fig. 1). We used urine specimens collected from $77 \mathrm{BC}$ patients and 136 healthy subjects (Table 2). We found that Bladder CARE effectively detects the presence of $\mathrm{BC}$ (both, high- and low-grade tumors; Table 4B) with an overall sensitivity of $93.5 \%$, a specificity of $92.6 \%$, and a PPV and NPV of $87.8 \%$ and $96.2 \%$, respectively (Table 3 ).

Within the control cohort we found 10 false-positive cases (Table $5 \mathrm{~A}$ ), potentially indicating that Bladder CARE may overestimate the presence of $\mathrm{BC}$. Interestingly, the majority of false-positive cases were found among the most at-risk populations for $\mathrm{BC}$ : males (7 out of 10 false-positive cases) [3], Caucasians (9 out of 10 false-positive cases) [1,2], and those between 50

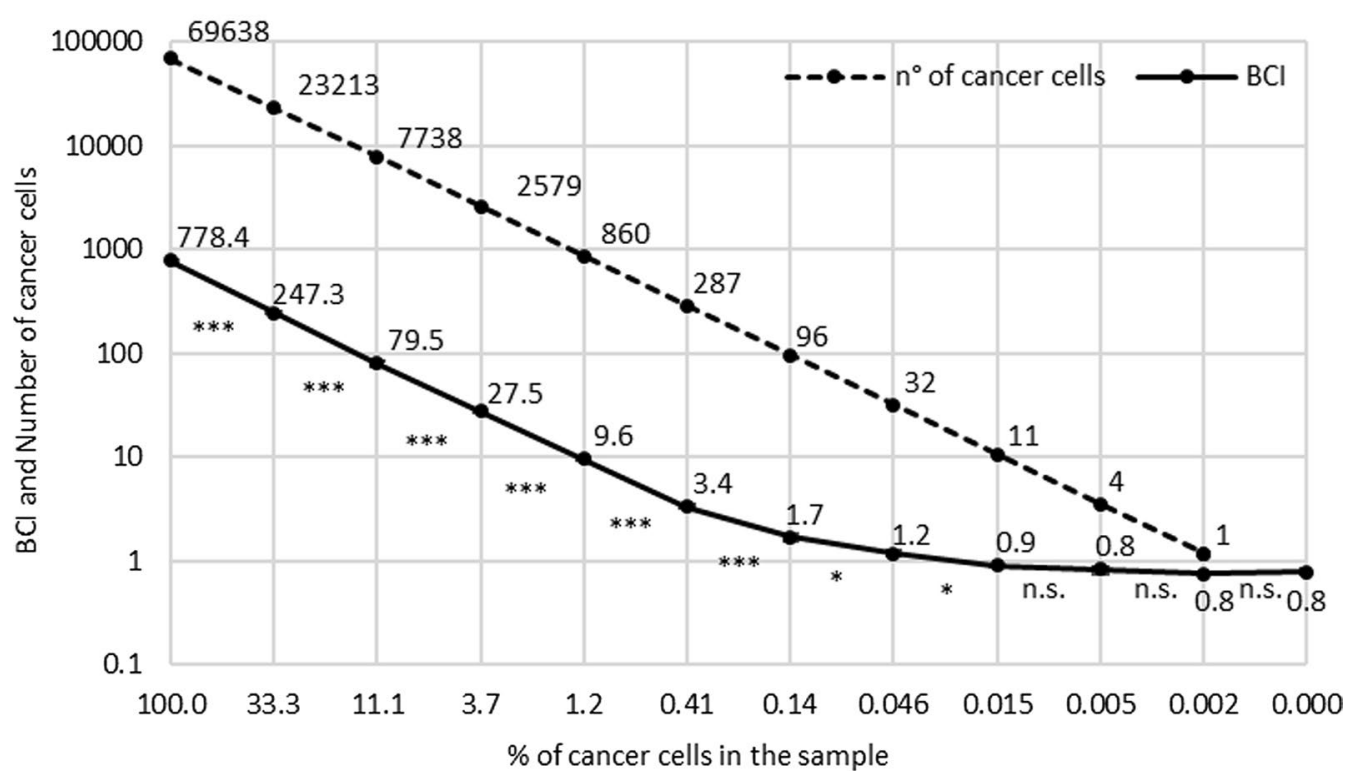

Fig. 5 Determination of Bladder CARE linearity and LOD. Bladder CARE linearity and LOD: comparison between the number of cancer cells (LD583 cancer cell line [41]) originally present in the 12 spike-in samples (dashed line and numbers above it) and the correspondent Bladder CARE Index (BCl) value (solid line and numbers above it). Each sample was tested using $500 \mathrm{ng}$ of spike-in DNA in three technical replicates. Significant differences in $\mathrm{BCl}$ between two consecutive samples are indicated by asterisks below the solid line. n.s. = not significant. Although barely visible, error bars for technical replicates are reported for each spike-in sample. Percentage of cancer cells in each spike-in sample (500 ng, correspondent to approximately 70,000 cells considering that one DNA molecule weighs $3.59 \mathrm{pg}$ [43]) is indicated in the $X$-axis. $\mathrm{BCl}$ value and number of cancer cells are represented in the $Y$-axis (logarithmic scale) 
and 80 years old (9 out of 10 false-positive cases) [1, 2] (Table 5B). It is possible that Bladder CARE detects early epigenetic alterations associated with pre-cancerous lesions which may not be yet associated with symptoms or a proper tumor formation. This may explain the apparent false-positive cases detected by Bladder CARE. However, one limitation of this study is that no clinical and follow-up information was available for the control cohort ("healthy" status was based on self-report); therefore, these suppositions are purely speculative, and more data need to be generated in the future order to better understand the reason for false-positive cases.

Within the cancer cohort we found 5 false-negative cases (Table 4A). A closer look at the data did not show any apparent correlation between false-negative cases and tumor type, grade, urine DNA yield, or presence of blood and leukocytes in the urine (Additional file 2: Table S3). Previously conducted interference experiments for urinary tract infections and hematuria reveal that these conditions do not interfere with Bladder CARE test results (data not shown). A possible explanation for false-negative cases is that the concentration of cancer cells in these samples may have been below Bladder CARE's LOD. Additional studies are required to fully elucidate this aspect.

Bladder CARE effectively detected the presence of both, high- and low-grade $\mathrm{BC}$ tumors with a sensitivity of $94.1 \%$ and $90.0 \%$, respectively (Table $4 \mathrm{~B}$ ). The high sensitivity of the test for both $\mathrm{BC}$ grades may be linked to the nature of epigenetic cancer biomarkers. Methylation status may become altered early during tumorigenesis and independently from the tumor stage or grade [39]. Although low-grade tumors rarely develop into aggressive and metastatic disease, their sensitive detection is still relevant from a diagnostic point of view since this type of tumor needs to be treated and patients followed to monitor for recurrence of the tumor. We must point out, however, that the number of low-grade $\mathrm{BC}$ samples included in this study is limited and future studies are necessary to confirm the highly sensitive detection of these type of tumors with Bladder CARE.

Urine samples for Bladder CARE are collected and stabilized at-home using the Bladder CARE Urine Collection Kit and can be mailed at ambient temperature [42]. None of the patients included in this study reported difficulties during remote sample collection, and all the samples received contain enough DNA to perform the Bladder CARE test. The remote sample collection and stabilization represents an important element of novelty in the field. It is advantageous for both the patient (especially for those that do not have easy access to the healthcare provider) and the physician since it can reduce otherwise unnecessary visits to the doctor office while still allowing close monitoring of the patients. Remote sample collection may also significantly lower the costs of surveillance and monitoring of $\mathrm{BC}$ patients.

We found that Bladder CARE has an LOD as low as 1 cancer cell for each 2,200 cells tested, and that the results are linear with the concentration of cancer cells present in the sample (Fig. 5, Additional file 2: Table S2). The low LOD may have important implications for early cancer detection. In addition, tumor signal can be monitored with Bladder CARE after TURBT or during treatments such as neoadjuvant chemotherapy in order to obtain information about the completeness of the resection procedure or the tumor response to treatments. This may allow urologists to intervene selectively and in a timely manner in cases where an increase of $\mathrm{BCI}$ is observed. We need to point out, however, that the LOD has been calculated in an artificial setting, using bladder cancer cell line DNA spiked in a background of genomic DNA; therefore, differences between the LOD reported in the manuscript and the true clinical LOD may exist.

We also provided evidence that bisulfite-free methods like Bladder CARE allow for maximal cancer signal in qPCR (Fig. 4) by preserving the integrity of cancerspecific methylated DNA regions. This is an advantage compared to methods that rely on bisulfite conversion of the DNA and which are known to cause significant DNA degradation $[31,38]$. Any loss of DNA (especially in samples with low and fragmented DNA like urine DNA and cell-free DNA) may have significant negative effects on the test result and may increase false-negative cases.

Finally, we also generated a model for estimating probability of cancer based on BCI results (Fig. 3a-c, Additional file 1: Figure S1). Knowing the probability of cancer in addition to Bladder CARE patient classification (Negative, High-Risk, and Positive) may further help a physician's decision making, especially for those patients with High-Risk Bladder CARE results.

\section{Conclusion}

Our study indicates that Bladder CARE is a good candidate for the simple, sensitive, and non-invasive detection of BC. While cystoscopy remains the gold standard to confirm the presence of $\mathrm{BC}$ and the first intervention procedure to remove $\mathrm{BC}$, Bladder CARE has the potential to reduce unnecessary cystoscopies and visits to the doctor office, allowing at the same time a close surveillance of $\mathrm{BC}$ patients. We envision that Bladder CARE, along with the Urine Collection Kit, could be used as a first-line test for the detection of $\mathrm{BC}$ and for the surveillance of those with a history of $\mathrm{BC}$. 


\section{Methods}

\section{Study population}

The study cohort consisted of patients with histologically confirmed urothelial carcinoma and healthy donors. The cancer cohort included specimens that were purchased from Geneticist Inc. (Glendale, CA, USA), and samples collected at the Department of Urology, USC Norris Comprehensive Cancer Center (Los Angeles, CA, USA), in accordance with an institutional review board approved protocol. The presence of $\mathrm{BC}$, as well as the tumor type and grade, was confirmed by cystoscopy and histology from resections or biopsies. No exclusion criteria were applied for BC patients. Samples from healthy donors were collected from consenting volunteers over 21 years old. "Healthy" status was based on self-reporting and defined as no history of any type of tumor. No follow-up information was collected for both cohorts.

\section{Urine sample collection, stabilization, and shipment}

Urine samples were collected and stabilized using the Bladder CARE Urine Collection Kit (Pangea Laboratory). No specific guidelines were established for the timing of urine sample collection. Samples purchased from Geneticist Inc. (Glendale, CA, USA) were analyzed with Multistix 10SG Urinalysis Test Strip (Siemens) prior to stabilization. Stabilized urine samples can be kept at room temperature for up to one month without DNA degradation or loss [42] and were mailed at ambient temperature to Pangea Laboratory for Bladder CARE analysis. A detailed description of the Bladder CARE workflow is presented in Fig. 1.

\section{Clinical procedures and Bladder CARE test}

Stabilized urine was processed and analyzed at Pangea Laboratory. After isolation using Quick-DNA ${ }^{\mathrm{TM}}$ Urine Kit (Zymo Research, D3061), urine DNA samples were quantified with Femto ${ }^{\mathrm{TM}}$ Human DNA Quantification Kit (Zymo Research, E2005). As low as 5 ng of urine DNA was analyzed in duplicate with Bladder CARE.

Briefly, the test measures the methylation level of three BC-specific biomarkers and two internal control loci (the last informing about the input DNA amount used in the test and the efficiency of the digestion step for each sample tested) using methylation-sensitive restriction enzymes coupled with qPCR [39]. Positive and negative
Bladder CARE control samples were also analyzed in parallel with clinical samples in order to confirm the validity of the test. A detailed description of the method is reported elsewhere [39, 40].

\section{Calculation of Bladder CARE test results (Bladder CARE Index-BCI)}

Bladder CARE results are expressed as the Bladder CARE Index $(\mathrm{BCI})$. $\mathrm{BCI}$ values are calculated by integrating the methylation level of the three $\mathrm{BC}$ biomarkers and the two internal controls in a proprietary algorithm developed by Pangea Laboratory [39, 40].

Based on BCI values, samples are categorized as 'Negative,' 'High-Risk', and 'Positive.' Specifically, samples with $\mathrm{BCI}<2.5$ are considered Negative for the presence of $\mathrm{BC}$, while samples with BCI between 2.5 and 5, and $>5$ are classified as High-Risk and Positive, respectively ([40], Fig. 1).

\section{Statistical analyses}

Results from a previous pilot study (57 healthy subjects and 51 BC patients; unpublished) were used to define each group's mean BCI values with 95\% CIs for the control and the cancer cohorts (control mean of 1.3 with a 95\% CI of 0.994 to 1.61 and cancer mean of 213.7 with a $95 \%$ CI of 86.7 to 341 ). We conducted a power analysis using the $n \_2 t$ unequal() function from the dvmisc package in $\mathrm{R}$, which allows group-specific variances (the cancer group has a much higher variance than control). Using the group-specific variances from the pilot data, an alpha threshold of 0.05 , and a difference of means of 200 BCI units, we established that we would need 45 samples per group to achieve $80 \%$ power in a comparison of control and cancer groups. In our study we included $77 \mathrm{BC}$ patients and 136 healthy subjects. Using the power_2t unequal() function we calculated that a sample size of 77 per group would result in $96.2 \%$ power at alpha of 0.05 to detect a difference in means of 200 BCI units.

Sensitivity, specificity, PPV and NPV of Bladder CARE were calculated based on the number of true-positive, true-negative, false-positive, and false-negative cases. All the samples classified as Negative by Bladder CARE $(\mathrm{BCI}<2.5)$ were considered negative in our study, while all the samples having a $\mathrm{BCI}>2.5$ (High-Risk and Positive Bladder CARE results) were considered positive. 
The statistical significance of differences in BCI values was determined using Student's two-tailed $t$ tests. Box plots of the cohorts by $\mathrm{BCI}$ values and the receiver operating characteristic (ROC) curve for the BCI classification (Fig. 2) were generated in python 3.7.2 using numpy 1.16 .2 and pandas 0.24 .2 packages for data processing, custom code to generate the true-positive rates and false-positive rates for the ROC curve sliding threshold, scikit-learn 0.20.3 to calculate the ROC area under the curve (AUC), and seaborn 0.9.0 with matplotlib 3.0.2 for visualization.

To provide an estimated probability of $\mathrm{BC}$ occurrence based on the BCI (Fig. 3, Additional file 1: Figure S1), a logistic regression model was developed utilizing BCI values and their corresponding clinical sample information (cystoscopy results and self-report). Logistic regression modeling was evaluated using a stratified $k$-fold cross-validation approach, where the dataset was divided into five similarly sized subsets containing a ratio of outcomes representative of the total dataset. Model selection and generation was then repeated five times (training with four of the subsets and testing with one), rotating the subset to be tested. Diagnostics measurements were generated for each fold, with the resulting model being chosen where the measurements most closely matched the mean of the cross-validation diagnostics. Calculations were performed in python 3.7.2 using the numpy 1.16.2 and pandas 0.24.2 packages for data processing, statsmodels 0.9 .0 for model generation, scikit-learn 0.20.3 for additional validation, and seaborn 0.9.0 with matplotlib 3.0.2 for visualization.

\section{Comparison between MSRE-qPCR and MS-qPCR}

Six standard DNA samples (800 ng each) were generated by combining different proportions of untreated and artificially methylated blood DNA (produced using M.SssI CpG Methylase; Zymo Research, E2011). Specifically, samples 1 to 6 contained 100\%, 33.3\%, 11.1\%, 3.7\%, $1.23 \%$, and $0.41 \%$, of artificially methylated DNA. In total, $400 \mathrm{ng}$ of each sample was bisulfite-treated using EZ DNA Methylation-Lightning Kit (Zymo Research, D5031), while the remaining $400 \mathrm{ng}$ was digested using $10 \mathrm{U}$ of the methylation-sensitive restriction enzyme HpaII (New England BioLabs, R0171S) and purified with DNA Clean and Concentrator-5 (Zymo Research,
D4013). Samples were then amplified by qPCR (CFX96 Touch qPCR System, Bio-Rad) using primers designed for bisulfite-converted methylated DNA (MS-qPCR) and genomic untreated DNA (MSRE-qPCR). Primers (sequences available upon request) have similar efficiencies and were designed on the same region of the $\mathrm{CpG}$ island of the human MGMT gene, which is known to be unmethylated in blood samples collected from healthy donors (Additional file 2: Table S1). Both amplicons have similar length and were amplified in triplicates in $20 \mu \mathrm{l}$ of reaction containing ZymoTaq qPCR Premix (Zymo Research, E2055), 0.4 $\mu \mathrm{M}$ of each primer, and the equivalent of $100 \mathrm{ng}$ of input DNA prior to bisulfite conversion or digestion. The program used to generate both amplicons has an initial 10 -min denaturation step at $95{ }^{\circ} \mathrm{C}$ followed by 40 cycles of denaturation at $97{ }^{\circ} \mathrm{C}$ for $20 \mathrm{~s}$ and annealing/extension at $58{ }^{\circ} \mathrm{C}$ for one minute. Data were analyzed using Bio-Rad CFX Maestro Software (Bio-Rad).

Methylation of the human MGMT target region in untreated and artificially methylated blood DNA was determined by MSRE-qPCR according to the protocol described above. Results are represented in Additional file 2: Table S1.

\section{LOD and linearity of Bladder CARE}

A set of 12 spike-in samples containing different amounts of LD583 (bladder carcinoma cell line [41]) DNA in a background of blood DNA isolated from a healthy donor were generated. Specifically, samples 1 to 12 contain $100 \%, 33.3 \%, 11.1 \%, 3.7 \%, 1.23 \%, 0.41 \%, 0.14 \%, 0.046 \%$, $0.015 \%, 0.005 \%, 0.0017 \%$, and 0\% of LD583 DNA, respectively (Additional file 2: Table S2). In total, $2 \mu \mathrm{g}$ of each sample was submitted to Pangea Laboratory and analyzed in triplicates (500 ng each) with Bladder CARE. The number of cancer cells originally used to generate the standards was calculated considering that a single human genomic DNA molecule weighs 3.59 pg [43]. For each sample, standard deviation and standard error were calculated based on the BCI values obtained from each of the three technical replicates. Student's two-tailed $t$ test was performed in order to determine the significance of BCI changes between consecutive samples (Additional file 2: Table S2). 


\section{Abbreviations}

BC: Bladder cancer; NMIBC: Non-muscle invasive bladder cancer; MIBC: Muscle invasive bladder cancer; TURBT: Transurethral resection of bladder tumor; MSRE-qPCR: Methylation-sensitive restriction enzyme-qPCR; MS-qPCR: Methylation-specific-qPCR

\section{Supplementary Information}

The online version contains supplementary material available at https://doi. org/10.1186/s13148-021-01029-1.

Additional file 1: Figure S1. Diagnostic Results of the Logistic Regression Model. Confusion matrix, prediction distributions, and receiver operating characteristic (ROC) curve of the chosen logistic regression model after cross-validation, visualizing the results of the test set (stratified $20 \%$ of total data, containing a representative ratio of the control/ cancer cohorts). (A) The area under the curve (AUC) of the ROC curve is a measure of model performance when considering model output as a classifier, plotting the difference in true positive rate (sensitivity) and false positive rate (1-specificity) as the classification decision boundary is changed (what threshold of percent probability is required to classify as positive), where $0.5=$ no discrimination and $1=$ perfect discrimination. (B) The model's probability outputs for the test set, with the $50 \%$ probability decision boundary shown as the dotted line. (C) Confusion Matrix outlining the model classification and true classification of the test set. Mean AUC from stratified $k$-fold cross-validation $(k=5)=0.974 \pm 0.02 \mathrm{Cl}$, with mean F1 score $=0.886 \pm 0.033 \mathrm{Cl}$. Chosen model Log Likelihood Ratio $p$ value $=1.047 \mathrm{e}-36$, with pseudo $R 2=0.715$

Additional file 2: Table S1. For each sample the percentage of methylation was calculated by applying the formula $2^{\wedge}-\Delta C T^{*} 100$, where $\Delta C T$ was calculated by subtracting the $\mathrm{CT}$ value of the Hpall restriction digestion reaction (Hpall $\mathrm{RD}$ ) from the $\mathrm{CT}$ value of the no restriction digestion reaction (No RD). The test was performed in two technical replicates. Table S2. Abbreviations: Rep, replicate; SD, standard deviation; SE, standard error; n.a., not applicable; LD583, cancer cell line [41]. * , number of cells (LD583 cancer cell line) are calculated considering that one DNA molecule weights 3.59 pg [43]. Table S3. Abbreviations: TCC, transitional cell carcinoma; n.a., not available. False negative cases are highlighted in grey. Blood and leukocytes contents was measured with Multistix 10SG Urinalysis Test Strips (Siemens), and results are reported based on manufacturer classification.

\section{Acknowledgements}

We would like to thank all the people that took part in this study. We are grateful to Dr. Jonathan Claypool (Zymo) for the extraordinary administrative and regulatory work done and Matt Grey (Pangea Laboratory) for contributing to the sample processing.

\section{Authors' contributions}

PP, YCC, XYJ, WG, GL helped in study concept and design. PP, YCC, GL, SG, SD, MS, BJ, TY, HA, FH, YT, YC acquired the data. PP, WG, JR, JMB, GL analyzed and interpreted the data. PP drafted the manuscript. SD, SG, GL, YCC helped in critical revision of the manuscript for important intellectual content. WG, PP, JR statistically analyzed the data. XYJ, GL, SD involved in obtaining funding. All authors helped in administrative, technical, or material support.

GL supervised the study. Others have nothing to disclose. All authors read and approved the final manuscript.

\section{Funding}

This research was coordinated by Zymo Research Corp. (Zymo) and by the Department of Urology, Keck School of Medicine, University of Southern California, Los Angeles, CA, USA. The research was sponsored through in-kind contributions (reagents and analyses necessary for the study) by Pangea Laboratory, LLC, and by Zymo. The study was supported by SC CTSI (received by G. Liang and S. Daneshmand), from Cure it Cancer Research (received by G. Liang and S. Daneshmand), and from The Vicky Joseph Cancer Research Foundation (received by G. Liang).

\section{Availability of data and materials}

The datasets used and/or analyzed during the current study are available from the corresponding author on reasonable request. G. Liang and P. Piatti have full access to all the data in the study and take responsibility for the integrity of the data and the accuracy of the data analysis.

\section{Ethics approval and consent to participate}

For the study, urine samples were collected (self-collection) from healthy subjects and patients affected by bladder cancer. Each healthy volunteer subject participating to this study signed a consent form provided by Zymo Research Corp. and approved by an internal committee. Urine from bladder cancer patients were collected in accordance with IRB-approved protocols at the Department of Urology, USC Norris Comprehensive Cancer Center (Los Angeles, CA, USA), and at Geneticist Inc. (Glendale, CA, USA).

\section{Consent for publication}

Not applicable.

\section{Competing interests}

G. Liang certifies that all conflicts of interest, including specific financial interests and relationships and affiliations relevant to the subject of matter or materials discussed in the manuscript (e.g., employment/affiliation, grants or funding, consultancies, honoraria, stock ownership or options, expert testimony, royalties, or patent applications that are pending or issued as patents), are the following: P. Piatti is named, along with W. Guo, X.Y. Jia (Jia), and G. Liang in US and foreign equivalent pending patent applications and issued patents for "Assays to Determine DNA Methylation and DNA Methylation Markers of Cancer" held by Zymo Research Corp. an associate of Pangea Laboratory, LLC. X.Y. Jia is an employee at Zymo Research Corp. and Pangea Laboratory, LLC. Dr. P. Piatti, Dr. Y.C. Chew, M. Suwoto, and T. Yamada are employees at Zymo Research Corp. and independent contractors with Pangea Laboratory, LLC. The remaining authors have nothing to disclose.

\section{Author details}

${ }^{1}$ Zymo Research Corp, Irvine, CA, USA. ${ }^{2}$ Pangea Laboratory LLC, Costa Mesa, CA, USA. ${ }^{3}$ Department of Urology, Keck School of Medicine, University of Southern California, Los Angeles, CA, USA.

Received: 16 September 2020 Accepted: 14 February 2021

Published online: 21 April 2021

\section{References}

1. Siegel RL, Miller KD, Jemal A. Cancer statistics, 2015. CA Cancer J Clin. 2015;65:5-29.

2. American Cancer Society. Cancer facts \& figures 2020. Atlanta: American Cancer Society; 2020.

3. Fajkovic H, Halpern JA, Cha EK, et al. Impact of gender on bladder cancer incidence, staging, and prognosis. World J Urol. 2011;29:457-63.

4. Freedman ND, Silverman DT, Hollenbeck AR, et al. Association between smoking and risk of bladder cancer among men and women. JAMA. 2011;306:737-45.

5. Branu S, Böhle A. Bladder cancer. I. molecular and genetic basis of carcinogenesis. Eur Urol. 2001;39:491-7.

6. Feng Z, Hu W, Rom WN, et al. 4-aminobiphenyl is a major etiological agent of human bladder cancer: evidence from its DNA binding spectrum in human p53 gene. Carcinogenesis. 2002;23:1721-7.

7. Samanic C, Kogevinas M, Dosemeci M, et al. Smoking and bladder cancer in Spain: effects of tobacco type, timing, environmental tobacco smoke, and gender. Cancer Epidemiol Biomark Prev. 2006;15:1348.

8. Abern MR, Dude AM, Tsivian M, et al. The characteristics of bladder cancer after radiotherapy for prostate cancer. Urol Oncol. 2013;31:1628.

9. Rothman N, Garcia-Closas M, Chatterjee N, et al. A multi-stage genomewide association study of bladder cancer identifies multiple susceptibility loci. Nat Genet. 2010;42:978.

10. Kaufman DS, Shipley WU, Feldman AS. Bladder cancer. Lancet. 2009:374:239-49.

11. Li HT, Duymich CE, Weisenberger DJ, et al. Genetic and epigenetic alterations in bladder cancer. Int Neurourol J. 2016;20(Suppl 2):S84-94. 
12. Babjuk M, Böhle A, Burger $M$, et al. EAU guidelines on non-muscleinvasive urothelial carcinoma of the bladder: update 2016. Eur Urol. 2016. https://doi.org/10.1016/j.eururo.2016.05.041.

13. Morgan TM, Keegan KA, Clark PE. Bladder cancer. Curr Opin Oncol. 2011;23:275-82.

14. Steinberg G. Blue light cystoscopy should be used routinely for bladder cancer detection: pro. J Urol. 2016;195(6):1652-3.

15. Svatek RS, Hollenbeck BK, Holmang S, et al. The economics of bladder cancer: costs and considerations of caring for this disease. Eur Urol. 2014;66:253-62

16. Gupta NP, Sharma N, Kumar R. Nuclear matrix protein 22 as adjunct to urine cytology and cystoscopy in follow-up of superficial TCC of urinary bladder. Urology. 2009;73:592-6.

17. Têtu B, Tiguert R, Harel F, et al. ImmunoCyt/uCyt+ improves the sensitivity of urine cytology in patients followed for urothelial carcinoma. Mod Pathol. 2005;18:83-9.

18. Irani J, Desgrandchamps F, Millet C, et al. BTA stat and BTA TRAK: a comparative evaluation of urine testing for the diagnosis of transitional cell carcinoma of the bladder. Eur Urol. 1999;35:89-92.

19. Sokolova IA, Halling KC, Jenkins RB, et al. The development of a multitarget, multicolor fluorescence in situ hybridization assay for the detection of urothelial carcinoma in urine. J Mol Diagn. 2000;2(3):116-23.

20. O'Sullivan P, Sharples K, Dalphin M, et al. A multigene urine test for the detection and stratification of bladder cancer in patients presenting with hematuria. J Urol. 2012;188(3):741-7.

21. Feber A, Dhami P, Dong L, et al. UroMark - a urinary biomarker assay for the detection of bladder cancer. Clin Epigenetics. 2017;9:8.

22. Witjes JA, Morote J, Cornel EB, et al. Performance of the bladder EpiCheck ${ }^{T M}$ methylation test for patients under surveillance for nonmuscle-invasive bladder cancer: results of a multicenter, prospective, blinded clinical trial. Eur Urol Oncol. 2018;1 (4):307-13.

23. Yafi FA, Brimo F, Steinberg J, et al. Prospective analysis of sensitivity and specificity of urinary cytology and other urinary biomarkers for bladder cancer. Urol Oncol. 2015;33(2):66e25-31.

24. Lotan Y, O'Sullivan P, Raman JD, et al. Clinical comparison of noninvasive urine tests for ruling out recurrent urothelial carcinoma. Urol Oncol. 2017;35(8):531e15-22.

25. Lozano Placio F, Morote J, Leibovitch I, et al. Performance of bladder EpiCheck ${ }^{\text {TM }}$ for NMIBC monitoring-updated results of a European multicenter study. Eur Urol Suppl. 2019;18(1):e947-9.

26. Mancini M, Righetto M, Zumerle S, et al. The bladder EpiCheck Test as a non-invasive tool based on the identification of DNA methylation in bladder cancer cells in the urine: a review of published evidence. Int J Mol Sci. 2020;21(18):6542.

27. Hajdinjak T. UroVysion FISH test for detecting urothelial cancers: metaanalysis of diagnostic accuracy and comparison with urinary cytology testing. Urol Oncol. 2008;26(6):646-51.
28. Lee J, Lee S, Kim W, et al. Efficacy and safety of hexaminolevulinate fluorescence cystoscopy in the diagnosis of bladder cancer. Korean J Urol. 2012:53:821-5.

29. Sharma S, Kelly TK, Jones PA. Epigenetics in cancer. Carcinogenesis. 2010:31:27-36.

30. Shen H, Laird PW. Interplay between the cancer genome and epigenome. Cell. 2013;153:38-55.

31. Locke WJ, Guanzon D, Ma C, et al. DNA methylation cancer biomarkers: translation to the clinic. Front Genet. 2019:10:1150.

32. Friedrich MG, Weisenberger DJ, Cheng JC, et al. Detection of methylated apoptosis associated genes in urine sediments of bladder cancer patients. Clin Cancer Res. 2004;10:7457-65.

33. Chihara $Y$, Kanai $Y$, Fujimoto $H$, et al. Diagnostic markers of urothelial cancer based on DNA methylation analysis. BMC Cancer. 2013;2013(13):275

34. Su SF, de Castro Abreu AL, Chihara Y, et al. A panel of three markers hyperand hypomethylated in urine sediments accurately predicts bladder cancer recurrence. Clin Cancer Res. 2014;20(7):1978-89.

35. Wolff EM, Byun HM, Han HF, et al. Hypomethylation of a LINE-1 promoter activates an alternate transcript of the MET oncogene in bladders with cancer. PLoS Genet. 2010;6(4):e1000917.

36. Dahl C, Guldberg P. DNA methylation analysis techniques. Biogerontology. 2003;4:233-50

37. Eads CA, Danenberg KD, Kawakami K, et al. Methylight: a high-throughput assay to measure DNA methylation. Nucleic Acids Res. 2000;28(8):E32.

38. Grunau C, Clark SJ, Rosenthal A. Bisulfite genomic sequencing: systematic investigation of critical experimental parameters. Nucleic Acids Res. 2001;29(13):E65-75.

39. Guo W, Piatti P, Yang X, et al. Assays to determine DNA methylation and DNA methylation markers of cancer. WO2016138105A2, 2016. https:// patents.google.com/patent/WO2016138105A2/ko.

40. Bladder CARE, Pangea Laboratory. https://www.pangealab.com/bladd ercare/.

41. Tsao JL, Yatabe Y, Markl I, et al. Bladder cancer genotype stability during clinical progression. Genes Chromosom Cancer. 2000;29:26-32.

42. Urine Collection Kit, Zymo Research. https://www.zymoresearch.com/ collections/urine-collection/products/urine-collection-kit.

43. Molecular Facts and Figures. IDT integraded DNA technologies. https:// sfvideo.blob.core.windows.net/sitefinity/docs/default-source/biotechbasics/molecular-facts-and-figures.pdf?sfvrsn $=4563407$ _4.

\section{Publisher's Note}

Springer Nature remains neutral with regard to jurisdictional claims in published maps and institutional affiliations.
Ready to submit your research? Choose BMC and benefit from:

- fast, convenient online submission

- thorough peer review by experienced researchers in your field

- rapid publication on acceptance

- support for research data, including large and complex data types

- gold Open Access which fosters wider collaboration and increased citations

- maximum visibility for your research: over $100 \mathrm{M}$ website views per year

At BMC, research is always in progress.

Learn more biomedcentral.com/submissions 